\title{
Iterative Algorithms for Variational Inequalities Governed by Boundedly Lipschitzian and Strongly Monotone Operators
}

\author{
Caiping Yang ${ }^{1}$ and Songnian $\mathrm{He}^{1,2}$ \\ ${ }^{1}$ College of Science, Civil Aviation University of China, Tianjin 300300, China \\ ${ }^{2}$ Tianjin Key Laboratory for Advanced Signal Processing, Civil Aviation University of China, Tianjin 300300, China \\ Correspondence should be addressed to Songnian He; songnianhe@163.com
}

Received 2 August 2014; Accepted 3 September 2014

Academic Editor: Wei-Shih Du

Copyright (c) 2015 C. Yang and S. He. This is an open access article distributed under the Creative Commons Attribution License, which permits unrestricted use, distribution, and reproduction in any medium, provided the original work is properly cited.

\begin{abstract}
Consider the variational inequality $\mathrm{VI}(C, F)$ of finding a point $x^{*} \in C$ satisfying the property $\left\langle F x^{*}, x-x^{*}\right\rangle \geq 0$ for all $x \in C$, where $C$ is a level set of a convex function defined on a real Hilbert space $H$ and $F: H \rightarrow H$ is a boundedly Lipschitzian (i.e., Lipschitzian on bounded subsets of $H$ ) and strongly monotone operator. He and Xu proved that this variational inequality has a unique solution and devised iterative algorithms to approximate this solution (see $\mathrm{He}$ and $\mathrm{Xu}, 2009$ ). In this paper, relaxed and self-adaptive iterative algorithms are proposed for computing this unique solution. Since our algorithms avoid calculating the projection $P_{C}$ (calculating $P_{C}$ by computing a sequence of projections onto half-spaces containing the original domain $C$ ) directly and select the stepsizes through a self-adaptive way (having no need to know any information of bounded Lipschitz constants of $F$ (i.e., Lipschitz constants on some bounded subsets of $H$ )), the implementations of our algorithms are very easy. The algorithms in this paper improve and extend the corresponding results of $\mathrm{He}$ and $\mathrm{Xu}$.
\end{abstract}

\section{Introduction}

Let $H$ be a real Hilbert space with inner product $\langle\cdot, \cdot\rangle$ and norm $\|\cdot\|$, let $C$ be a nonempty closed convex subset of $H$, and let $F: C \rightarrow H$ be a nonlinear operator. We consider the problem of finding a point $x^{*} \in C$ with the property

$$
\left\langle F x^{*}, x-x^{*}\right\rangle \geq 0, \quad \forall x \in C .
$$

This is known as the variational inequality problem $\mathrm{VI}(C, F)$, initially introduced and studied by Stampacchia [1] in 1964. In recent years, variational inequality problems have been extended to study a large variety of problems arising in structural analysis, economics, optimization, operations research, and engineering sciences; see [1-21] and the references therein. Using the projection technique, one can easily show that $\operatorname{VI}(C, F)$ is equivalent to a fixed-point problem (see, e.g., [15]).

Lemma 1. $x^{*} \in C$ is a solution of $\operatorname{VI}(C, F)$ if and only if $x^{*} \in C$ satisfies the fixed-point relation:

$$
x^{*}=P_{C}(I-\lambda F) x^{*},
$$

where $\lambda>0$ is an arbitrary constant, $P_{C}$ is the orthogonal projection onto $C$, and $I$ is the identity operator on $H$.

Recall that an operator $F: C \rightarrow H$ is called monotone, if

$$
\langle F x-F y, x-y\rangle \geq 0 \quad \forall x, y \in C .
$$

Moreover, a monotone operator $F$ is called strictly monotone if the equality " $=$ " holds only when $x=y$ in the last relation. It is easy to see that $\operatorname{VI}(C, F)(1)$ has at most one solution if $F$ is strictly monotone.

For variational inequality (1), $F$ is generally assumed to be Lipschitzian and strongly monotone on $C$; that is, for some constants $\kappa, \eta>0, F$ satisfies the conditions

$$
\begin{gathered}
\|F x-F y\| \leq \kappa\|x-y\|, \quad \forall x, y \in C, \\
\langle F x-F y, x-y\rangle \geq \eta\|x-y\|^{2}, \quad \forall x, y \in C .
\end{gathered}
$$

In this case, $F$ is also called a $\kappa$-Lipschitzian and $\eta$-strongly monotone operator. It is not difficult to show the following result. 
Lemma 2. Assume that $F$ satisfies conditions (4) and (5) and $\lambda$ and $\mu$ are constants such that $\lambda \in(0,1)$ and $\mu \in\left(0,2 \eta / \kappa^{2}\right)$, respectively. Let $T^{\mu}=P_{C}(I-\mu F)($ or $I-\mu F)$ and $T^{\lambda, \mu}=P_{C}(I-$ $\lambda \mu F)($ or $I-\lambda \mu F)$. Then $T^{\mu}$ and $T^{\lambda, \mu}$ are all contractions with coefficients $1-\tau$ and $1-\lambda \tau$, respectively, where $\tau=(1 / 2) \mu(2 \eta-$ $\left.\mu L^{2}\right)$.

By using the well-known Banach contraction mapping principle, this fact together with Lemma 1 leads to the following classical result.

Theorem 3. Assume that $F$ satisfies conditions (4) and (5). Then $\operatorname{VI}(C, F)$ has a unique solution. Moreover, for any $0<$ $\lambda<2 \eta / \kappa^{2}$, the sequence $\left\{x_{n}\right\}$ with initial guess $x_{0} \in C$ and defined recursively by

$$
x_{n+1}=P_{C}(I-\lambda F) x_{n}, \quad n \geq 0,
$$

converges strongly to the unique solution of $\operatorname{VI}(C, F)$.

Attempts are worth making to weaken the Lipschitz condition (4) or the strong monotonicity condition (5) so that existence of solutions of variational inequality (1) is still guaranteed. In 2009, $\mathrm{He}$ and $\mathrm{Xu}$ [14] weakened the Lipschitz condition (4) successfully to the bounded Lipschitz condition. A mapping $F: C \rightarrow H$ is boundedly Lipschitzian on $C$ if it is Lipschitzian on each bounded subset of $C$; namely, for each nonempty bounded subset $B$ of $C$, there exists a positive constant $\kappa_{B}$ depending only on the set $B$ such that

$$
\|F x-F y\| \leq \kappa_{B}\|x-y\|, \quad \forall x, y \in B .
$$

$\mathrm{He}$ and $\mathrm{Xu}[14]$ not only proved existence and uniqueness of solutions of $\operatorname{VI}(C, F)$ under conditions (5) and (7) but also estimated the range of this unique solution.

Theorem 4 (see [14]). Assume that F :C $\rightarrow H$ is boundedly Lipschitzian on $C$ (i.e., for each bounded subset $B$ of $C, F$ is Lipschitzian on $B$ ). Assume also that $F$ is $\eta$-strongly monotone on $C$. Then variational inequality (1) has a unique solution $x^{*} \in C$ such that

$$
\left\|x^{*}-u\right\| \leq \frac{1}{\eta}\|F u\|
$$

where $u \in C$ is an arbitrary fixed element.

Similarly, we can also introduce bounded strong monotonicity of an operator. An operator $F: C \rightarrow H$ is called boundedly strong monotone on $C$, if, for arbitrary bounded subset $B$ of $C$, there exists a positive constant $\eta_{B}$ depending only on the set $B$ such that

$$
\langle F(x)-F(y), x-y\rangle \geq \eta_{B}\|x-y\|^{2}, \quad \forall x, y \in B .
$$

So a natural question gives rise to this: is it possible also to replace the strong monotonicity of $F$ by bounded strong monotonicity so that the result of Theorem 4 is still guaranteed? Unfortunately, a simple example [14] gives us a negative answer.
$\mathrm{He}$ and $\mathrm{Xu}[14]$ also consider the iterative algorithms for solving $\operatorname{VI}(C, F)$, where $F: C \rightarrow H$ is boundedly Lipschitzian and $\eta$-strongly monotone on $C$. Denote by $u$ an arbitrary fixed element in $C$ and denote by $r$ a positive fixed constant such that

$$
r \geq \frac{1}{\eta}\|F u\| .
$$

Set $C_{r}=S(u, r) \cap C(S(u, r)$ is a closed ball of $H$, i.e., $S(u, r)=$ $\{x \in H:\|x-u\| \leq r\})$ and denote by $\kappa_{r}$ the Lipschitz constant of $F$ on the bounded closed convex subset $C_{r}$.

Using Theorem 4, it is easy to see that $\mathrm{VI}(C, F)$ and $\operatorname{VI}\left(C_{r}, F\right)$ have the same solution. Thus one can devise iterative methods for $\operatorname{VI}\left(C_{r}, F\right)$ and get the unique solution of $\mathrm{VI}(C, F)$.

Theorem 5 (see [14]). Define a sequence $\left\{x_{n}\right\}$ recursively by the iterative algorithm

$$
\begin{gathered}
x_{0} \in C_{r} \text { arbitrarily, } \\
x_{n+1}=P_{C_{r}}(I-\lambda F) x_{n},
\end{gathered}
$$

where $0<\lambda<2 \eta / \kappa_{r}^{2}$. Then $\left\{x_{n}\right\}$ converges strongly to the unique solution $x^{*}$ of $\operatorname{VI}(C, F)$.

However, algorithms (6) and (11) all have two evident weaknesses. On the one hand, they involve calculating the projections $P_{C}$ and $P_{C_{r}}$, respectively, while the computation of a projection onto a closed convex subset is generally difficult. Particularly, the computation of $P_{C_{r}}$ is maybe more difficult since the structure of $C_{r}$ is more complicated. On the other hand, the determination of the stepsize $\lambda$ depends on the constants $\kappa$ (or $\kappa_{r}$ ) and $\eta$. This means that, in order to implement algorithm (6) (or algorithm (11)), one has first to compute (or estimate) the constants $\kappa$ (or $\kappa_{r}$ ) and $\eta$, which is sometimes not an easy work in practice.

$\mathrm{He}$ and Yang [22] proposed relaxed and self-adaptive algorithms in order to overcome the above weaknesses of algorithm (6) and proved strong convergence theorems.

In order to overcome the above weaknesses of algorithm (11), new relaxed and self-adaptive algorithms are proposed in this paper to solve $\operatorname{VI}(C, F)$, where $C$ is a level set of a convex function defined on $H$ and $F: H \rightarrow H$ is a boundedly Lipschitzian and $\eta$-strongly monotone operator. Our methods calculate $P_{C_{r}}$ by computing $P_{S(u, r)}$ (the computation of $P_{S(u, r)}$ is very easy) and a sequence of projections onto half-spaces containing the original level set $C$ and select the stepsizes through a self-adaptive way. The implementations of our algorithms are very easy since they avoid computing $P_{C_{r}}$ directly and have no need to know any information about $\kappa_{r}$ (but $\eta$ is assumed to be known, so our methods partly overcome the second weakness above). The algorithms in this paper improve and extend the above corresponding result of $\mathrm{He}$ and $\mathrm{Xu}$.

The rest of this paper is organized as follows. Some useful lemmas are listed in the next section. In the last section, a relaxed algorithm for the case where $\eta$ and $\kappa_{r}$ are all known and a relaxed self-adaptive algorithm for the case where $\eta$ 
is known but $\kappa_{r}$ is unknown are proposed, respectively. The strong convergence theorems of our algorithms are proved.

\section{Preliminaries}

Throughout the rest of this paper, we denote by $H$ a real Hilbert space and by $I$ the identity operator on $H$. If $f: H \rightarrow$ $\mathbb{R}$ is a differentiable functional, then we denote by $\nabla f$ the gradient of $f$. We will also use the following notations.

(i) $\rightarrow$ denotes strong convergence.

(ii) $\rightarrow$ denotes weak convergence.

(iii) $S(u, r)=\{x \mid\|x-u\| \leq r\}$ denotes a closed ball in $H$ with center $u$ and radius $r$.

(iv) $\omega_{w}\left(x_{n}\right)=\left\{x \mid \exists\left\{x_{n_{k}}\right\} \subset\left\{x_{n}\right\}\right.$ such that $\left.x_{n_{k}} \rightarrow x\right\}$ denotes the weak $\omega$-limit set of $\left\{x_{n}\right\}$.

Recall a trivial inequality, which is well known and in common use.

Lemma 6. For all $x, y \in H$, there holds the relation

$$
\|x+y\|^{2} \leq\|x\|^{2}+2\langle y, x+y\rangle .
$$

Recall that a mapping $T: H \rightarrow H$ is said to be nonexpansive if

$$
\|T x-T y\| \leq\|x-y\|, \quad x, y \in H .
$$

$T: H \rightarrow H$ is said to be firmly nonexpansive if, for $x, y \in H$,

$$
\|T x-T y\|^{2} \leq\|x-y\|^{2}-\|(I-T) x-(I-T) y\|^{2} .
$$

The following are characterizations of firmly nonexpansive mappings (see [7] or [23]).

Lemma 7. Let $T: H \rightarrow H$ be an operator. The following statements are equivalent.

(i) $T$ is firmly nonexpansive.

(ii) $I-T$ is firmly nonexpansive.

(iii) $\|T x-T y\|^{2} \leq\langle x-y, T x-T y\rangle, x, y \in H$.

We know that the orthogonal projection $P_{C}$ from $H$ onto a nonempty closed convex subset $C \subset H$ is a typical example of a firmly nonexpansive mapping, which is defined by

$$
P_{C} x:=\arg \min _{y \in C}\|x-y\|^{2}, \quad x \in H .
$$

It is well known that $P_{C} x$ is characterized by the inequality (for $x \in H$ )

$$
P_{C} x \in C, \quad\left\langle x-P_{C} x, y-P_{C} x\right\rangle \leq 0, \quad \forall y \in C .
$$

The following recent result [22] is likely to become a new fundamental tool for proving strong convergence of some algorithms. Its key effect on the proofs of our main results will be illustrated in the next section.
Lemma 8 (see [22]). Assume $s_{n}$ is a sequence of nonnegative real numbers such that

$$
\begin{gathered}
s_{n+1} \leq\left(1-\gamma_{n}\right) s_{n}+\gamma_{n} \delta_{n}, \quad n \geq 0, \\
s_{n+1} \leq s_{n}-\eta_{n}+\alpha_{n}, \quad n \geq 0,
\end{gathered}
$$

where $\left(\gamma_{n}\right)$ is a sequence in $(0,1),\left(\eta_{n}\right)$ is a sequence of nonnegative real numbers, and $\left(\delta_{n}\right)$ and $\left(\alpha_{n}\right)$ are two sequences in $\mathbb{R}$ such that

(i) $\sum_{n=0}^{\infty} \gamma_{n}=\infty$,

(ii) $\lim _{n \rightarrow \infty} \alpha_{n}=0$,

(iii) $\lim _{k \rightarrow \infty} \eta_{n_{k}}=0$ implies $\lim \sup _{k \rightarrow \infty} \delta_{n_{k}} \leq 0$ for any subsequence $\left(n_{k}\right) \subset(n)$.

Then $\lim _{n \rightarrow \infty} s_{n}=0$.

Recall that a function $f: H \rightarrow \mathbb{R}$ is called convex if

$$
\begin{aligned}
f(\lambda x+(1-\lambda) y) & \leq \lambda f(x)+(1-\lambda) f(y), \\
& \forall \lambda \in(0,1), \quad \forall x, y \in H .
\end{aligned}
$$

A differentiable function $f$ is convex if and only if there holds the relation

$$
f(z) \geq f(x)+\langle\nabla f(x), z-x\rangle, \quad \forall z \in H .
$$

Recall that an element $g \in H$ is said to be a subgradient of $f: H \rightarrow \mathbb{R}$ at $x$ if

$$
f(z) \geq f(x)+\langle g, z-x\rangle, \quad \forall z \in H .
$$

A function $f: H \rightarrow \mathbb{R}$ is said to be subdifferentiable at $x$, if it has at least one subgradient at $x$. The set of subgradients of $f$ at the point $x$ is called the subdifferential of $f$ at $x$ and is denoted by $\partial f(x)$. The last relation above is called the subdifferential inequality of $f$ at $x$. A function $f$ is called subdifferentiable, if it is subdifferentiable at all $x \in H$. If a function $f$ is differentiable and convex, then its gradient and subgradient coincide.

Recall that a function $f: H \rightarrow \mathbb{R}$ is said to be weakly lower semicontinuous $(w-l s c)$ at $x$ if $x_{n} \rightarrow x$ implies

$$
f(x) \leq \liminf _{n \rightarrow \infty} f\left(x_{n}\right) .
$$

\section{Iterative Algorithms}

In this section, we consider the iterative algorithms for solving a particular kind of variational inequality (1) in which the closed convex subset $C$ is of the particular structure, that is, the level set of a convex function given as follows:

$$
C=\{x \in H: c(x) \leq 0\},
$$

where $c: H \rightarrow \mathbb{R}$ is a convex function. We always assume that $c$ is subdifferentiable on $H$ and $\partial c$ is bounded operator (i.e., bounded on bounded sets). We also assume that $F: H \rightarrow H$ is a boundedly Lipschitzian and $\eta$-strongly monotone operator. Using Theorem 4, we assert that in this 
case $\operatorname{VI}(C, F)$ has a unique solution, henceforth, which is denoted by $x^{*}$.

The computation of a projection onto a closed convex subset is generally difficult. To overcome this difficulty, Fukushima [21] suggested a way to calculate the projection onto a level set of a convex function by computing a sequence of projections onto half-spaces containing the original level set. This idea is followed by Yang [24] and Lopez et al. and so forth [25], respectively, who introduced the relaxed CQ algorithms for solving the split feasibility problem in a finitedimensional and infinite-dimensional Hilbert space, respectively. He and Yang [22] also used this idea to devise iterative algorithms for solving variational inequalities governed by Lipschitzian and strongly monotone operators.

In the sequel, we always assume that $\eta$ is known and denote by $x_{0} \in H$ a selected arbitrarily fixed element. Using Theorem 4 , the unique solution $x^{*}$ of $\operatorname{VI}(C, F)$ belongs to a closed ball $S\left(x_{0}, r\right)$, where $r$ is a fixed positive constant such that $r>\left\|F x_{0}\right\| / \eta$. We also always denote by $\kappa_{r}$ the Lipschitz constant of $F$ on $S\left(x_{0}, r\right)$.

Based on Theorem 4, we are now in a position to introduce a relaxed algorithm for computing the unique solution $x^{*}$ of $\operatorname{VI}(C, F)$, where $C$ is given as in (23). This scheme applies to the case where $\kappa_{r}$ is easy to be determined.

Algorithm 9. Choose an arbitrary initial guess $x_{0} \in H$ and the sequence $\left(x_{n}\right)$ is constructed via the formula

$$
x_{n+1}=P_{S\left(x_{0}, r\right)} P_{C_{n}}\left(I-\lambda_{n} \mu_{r} F\right) x_{n}, \quad n \geq 0,
$$

where

$$
C_{n}=\left\{x \in H: c\left(x_{n}\right) \leq\left\langle\xi_{n}, x_{n}-x\right\rangle\right\},
$$

where $\xi_{n} \in \partial c\left(x_{n}\right)$, the sequence $\left(\lambda_{n}\right)$ in $(0,1)$, and $\mu_{r}$ is a constant such that $\mu_{r} \in\left(0,2 \eta / \kappa_{r}^{2}\right)$.

We now analyze strong convergence of Algorithm 9, which also illustrates the application of Lemma 8.

Theorem 10. Assume that $\lambda_{n} \rightarrow 0(n \rightarrow \infty)$ and $\sum_{n=1}^{+\infty} \lambda_{n}=$ $+\infty$. Then the sequence $\left(x_{n}\right)$ generated by Algorithm 9 converges strongly to the unique solution $x^{*}$ of $\operatorname{VI}(C, F)$.

Proof. Obviously, it follows from (24) that $\left(x_{n}\right)$ is bounded (indeed $\left.\left(x_{n}\right) \subset S\left(x_{0}, r\right)\right)$ and so is $\left(F x_{n}\right)$ noting the bounded Lipschitz condition of $F$. It is easy to see from the subdifferential inequality and the definition of $C_{n}$ that $C_{n} \supset C$ holds for all $n \geq 0$, and hence it follows that $S\left(x_{0}, r\right) \cap C_{n} \supset S\left(x_{0}, r\right) \cap C=$ $C_{r}$. Observing that $x^{*} \in C_{r}$, a projection is nonexpansive, and $T^{\lambda_{n}}=I-\lambda_{n} \mu_{r} F: S\left(x_{0}, r\right) \rightarrow H$ is a contraction with coefficient $1-\lambda_{n} \tau_{r}$ for all $n \geq 0$ (using Lemma 2), where $\tau_{r}=(1 / 2) \mu_{r}\left(2 \eta-\mu_{r} \kappa_{r}^{2}\right)$, we obtain using (24) and Lemma 6 that

$$
\begin{aligned}
& \left\|x_{n+1}-x^{*}\right\|^{2} \\
& \quad=\left\|P_{S\left(x_{0}, r\right)} P_{C_{n}}\left(I-\lambda_{n} \mu_{r} F\right) x_{n}-P_{S\left(x_{0}, r\right)} P_{C_{n}} x^{*}\right\|^{2} \\
& \quad \leq\left\|\left(I-\lambda_{n} \mu_{r} F\right) x_{n}-\left(I-\lambda_{n} \mu_{r} F\right) x^{*}-\lambda_{n} \mu_{r} F x^{*}\right\|^{2}
\end{aligned}
$$

$$
\begin{aligned}
\leq & \left(1-\tau_{r} \lambda_{n}\right)\left\|x_{n}-x^{*}\right\|^{2}-2 \lambda_{n} \mu_{r}\left\langle F x^{*}, x_{n}-x^{*}-\lambda_{n} \mu_{r} F x_{n}\right\rangle \\
\leq & \left(1-\tau_{r} \lambda_{n}\right)\left\|x_{n}-x^{*}\right\|^{2}-2 \lambda_{n} \mu_{r}\left\langle F x^{*}, x_{n}-x^{*}\right\rangle \\
& +2 \lambda_{n}^{2} \mu_{r}^{2}\left\|F x^{*}\right\|\left\|F x_{n}\right\| .
\end{aligned}
$$

On the other hand, we also have

$$
\begin{aligned}
& \left\|x_{n+1}-x^{*}\right\|^{2} \\
& =\left\|P_{S\left(x_{0}, r\right)} P_{C_{n}}\left(I-\lambda_{n} \mu_{r} F\right) x_{n}-P_{S\left(x_{0}, r\right)} P_{C_{n}} x^{*}\right\|^{2} \\
& =\| P_{S\left(x_{0}, r\right)} P_{C_{n}}\left(I-\lambda_{n} \mu_{r} F\right) x_{n}-P_{S\left(x_{0}, r\right)} P_{C_{n}} x_{n} \\
& \quad+P_{S\left(x_{0}, r\right)} P_{C_{n}} x_{n}-P_{S\left(x_{0}, r\right)} P_{C_{n}} x^{*} \|^{2} \\
& \leq\left\|P_{S\left(x_{0}, r\right)} P_{C_{n}} x_{n}-P_{S\left(x_{0}, r\right)} P_{C_{n}} x^{*}\right\|^{2} \\
& \quad+2 \lambda_{n} \mu_{r}\left\|F x_{n}\right\| \cdot\left\|x_{n}-x^{*}\right\|+\lambda_{n}^{2} \mu_{r}^{2}\left\|F x_{n}\right\|^{2} \\
& \leq\left\|P_{S\left(x_{0}, r\right)} P_{C_{n}} x_{n}-P_{S\left(x_{0}, r\right)} P_{C_{n}} x^{*}\right\|^{2}+\lambda_{n} M,
\end{aligned}
$$

where $M$ is a positive constant such that $M \geq \sup _{n}\left\{2 \mu_{r}\left\|F x_{n}\right\| \cdot\right.$ $\left.\left\|x_{n}-x^{*}\right\|+\lambda_{n} \mu_{r}^{2}\left\|F x_{n}\right\|^{2}\right\}$. Observing that a projection is firmly nonexpansive, we have

$$
\begin{aligned}
& \left\|P_{S\left(x_{0}, r\right)} P_{C_{n}} x_{n}-P_{S\left(x_{0}, r\right)} P_{C_{n}} x^{*}\right\|^{2} \\
& \quad \leq\left\|P_{C_{n}} x_{n}-P_{C_{n}} x^{*}\right\|^{2}-\left\|P_{C_{n}} x_{n}-P_{S\left(x_{0}, r\right)} P_{C_{n}} x_{n}\right\|^{2} \\
& \quad \leq\left\|x_{n}-x^{*}\right\|^{2}-\left\|x_{n}-P_{C_{n}} x_{n}\right\|^{2}-\left\|P_{C_{n}} x_{n}-P_{S\left(x_{0}, r\right)} P_{C_{n}} x_{n}\right\|^{2},
\end{aligned}
$$

and the combination of (27) and (28) leads to

$$
\begin{aligned}
\left\|x_{n+1}-x^{*}\right\|^{2} \leq & \left\|x_{n}-x^{*}\right\|^{2}-\left\|x_{n}-P_{C_{n}} x_{n}\right\|^{2} \\
& -\left\|P_{C_{n}} x_{n}-P_{S\left(x_{0}, r\right)} P_{C_{n}} x_{n}\right\|^{2}+\lambda_{n} M .
\end{aligned}
$$

Setting

$$
\begin{gathered}
s_{n}=\left\|x_{n}-x^{*}\right\|^{2}, \quad \gamma_{n}=\tau_{r} \lambda_{n}, \\
\delta_{n}=-\frac{2 \mu_{r}}{\tau_{r}}\left\langle F x^{*}, x_{n}-x^{*}\right\rangle+2 \lambda_{n} \frac{\mu_{r}^{2}}{\tau_{r}}\left\|F x^{*}\right\|\left\|F x_{n}\right\|, \\
\eta_{n}=\left\|x_{n}-P_{C_{n}} x_{n}\right\|^{2}+\left\|P_{C_{n}} x_{n}-P_{S\left(x_{0}, r\right)} P_{C_{n}} x_{n}\right\|^{2}, \\
\alpha_{n}=M \lambda_{n},
\end{gathered}
$$

then (26) and (29) can be rewritten as the following forms, respectively:

$$
\begin{gathered}
s_{n+1} \leq\left(1-\gamma_{n}\right) s_{n}+\gamma_{n} \delta_{n}, \\
s_{n+1} \leq s_{n}-\eta_{n}+\alpha_{n} .
\end{gathered}
$$


Since $\sum_{n=1}^{\infty} \gamma_{n}=\infty$ and $\alpha_{n} \rightarrow 0$ hold, in order to complete the proof using Lemma 8 , it suffices to verify that

$$
\lim _{k \rightarrow \infty} \eta_{n_{k}}=0
$$

implies

$$
\limsup _{k \rightarrow \infty} \delta_{n_{k}} \leq 0
$$

for any subsequence $\left(n_{k}\right) \subset(n)$. In fact, if $\eta_{n_{k}} \rightarrow 0$ as $k \rightarrow \infty$, then $\left\|x_{n_{k}}-P_{C_{n_{k}}} x_{n_{k}}\right\| \rightarrow 0$ and $\| P_{C_{n_{k}}} x_{n_{k}}-$ $P_{S\left(x_{0}, r\right)} P_{C_{n_{k}}} x_{n_{k}} \| \rightarrow 0$ hold. Since $\partial c$ is bounded on bounded sets, we have a positive constant $\sigma$ such that $\left\|\xi_{n_{k}}\right\| \leq \sigma$ for all $k \geq 0$. From (25) and the trivial fact that $P_{C_{n_{k}}} x_{n_{k}} \in C_{n_{k}}$, it follows that

$$
c\left(x_{n_{k}}\right) \leq\left\langle\xi_{n_{k}}, x_{n_{k}}-P_{C_{n_{k}}} x_{n_{k}}\right\rangle \leq \sigma\left\|x_{n_{k}}-P_{C_{n_{k}}} x_{n_{k}}\right\| .
$$

Take $x^{\prime} \in \omega_{w}\left(x_{n_{k}}\right)$ arbitrarily and assume that $x_{n_{k}} \rightarrow x^{\prime}$ without loss of the generality; then the $w-l s c$ of $c$ and (35) imply that

$$
c\left(x^{\prime}\right) \leq \liminf _{k \rightarrow \infty} c\left(x_{n_{k}}\right) \leq 0 .
$$

This means that $x^{\prime} \in C$ holds. On the other hand, we assert from $\left(x_{n}\right) \subset S\left(x_{0}, r\right)$ that $x^{\prime} \in S\left(x_{0}, r\right)$. Moreover, we obtain that $x^{\prime} \in S\left(x_{0}, r\right) \cap C=C_{r}$ and hence $\omega_{w}\left(x_{n_{k}}\right) \subset C_{r}$.

Noting the fact that $2 \lambda_{n}\left(\mu_{r}^{2} / \tau_{r}\right)\left\|F x^{*}\right\|\left\|F x_{n}\right\| \rightarrow 0(n \rightarrow$ $\infty)$ and $x^{*}$ is the unique solution of $\operatorname{VI}\left(C_{r}, F\right)$ (i.e., the unique solution of $\operatorname{VI}(C, F))$, it turns out that

$$
\begin{aligned}
\limsup _{k \rightarrow \infty} \delta_{n_{k}} & =-\frac{2 \mu}{\tau} \liminf _{k \rightarrow \infty}\left\langle F x^{*}, x_{n_{k}+1}-x^{*}\right\rangle \\
& \leq-\frac{2 \mu}{\tau} \liminf _{k \rightarrow \infty}\left\langle F x^{*}, x_{n_{k}}-x^{*}\right\rangle \\
& =-\frac{2 \mu}{\tau} \inf \left\langle F x^{*}, w-x^{*}\right\rangle \leq 0,
\end{aligned}
$$

$w \in \omega_{w}\left(x_{n_{k}}\right)$.

It is worth mentioning that if $P_{C}$ is easy to be calculated, then $P_{C_{n}}$ in Algorithm 9 can be replaced with $P_{C}$ and it is easy to see that the whole proof of Theorem 10 is valid for this case. Therefore, if $C=H, \operatorname{VI}(C, F)$ reduces to the operator equation problem: finding $x^{*} \in H$ such that $F x^{*}=0$, and the following result holds.

Corollary 11. Assume that $\lambda_{n} \rightarrow 0(n \rightarrow \infty), \sum_{n=1}^{+\infty} \lambda_{n}=$ $+\infty$, and $C=H$. Then the sequence $\left(x_{n}\right)$ generated by algorithm

$$
x_{n+1}=P_{S\left(x_{0}, r\right)}\left(I-\lambda_{n} \mu_{r} F\right) x_{n}, \quad n \geq 0
$$

converges strongly to the unique solution $x^{*}$ of the operator equation $F x=0$.
Sometimes, the constant $\kappa_{r}$ is difficult to be obtained or estimated in practice (but we assume that $\eta$ has been obtained). In this case, Algorithm 9 is indeed not fit for solving $\operatorname{VI}\left(C_{r}, F\right)$ (i.e., $\operatorname{VI}(C, F)$ ). Then we now turn to introducing a relaxed and self-adaptive algorithm for the case where constant $\kappa_{r}$ is unknown.

Algorithm 12. Choose an arbitrary initial guess $x_{0} \in C$ and an arbitrary element $x_{1} \in S\left(x_{0}, r\right)$ such that $x_{1} \neq x_{0}$. Assume that the $n$th iterate $x_{n}(n \geq 1)$ has been constructed. Continue and calculate the $(n+1)$ th iterate $x_{n+1}$ via the formula

$$
x_{n+1}=P_{S\left(x_{0}, r\right)} P_{C_{n}}\left(I-\lambda_{n} \mu_{n} F\right) x_{n}, \quad n \geq 1,
$$

where $C_{n}$ is given as in (25), the sequence $\left(\lambda_{n}\right)$ is in $(0,1), r$ is a constant such that $r>\left\|F x_{0}\right\| / \eta$, and the sequence $\left(\mu_{n}\right)$ is determined via the relation

$$
\mu_{n}=\left\{\begin{array}{lr}
\frac{\eta\left\|x_{n}-x_{n-1}\right\|^{2}}{\left\|F x_{n}-F x_{n-1}\right\|^{2}}, & \text { if } x_{n} \neq x_{n-1}, \\
\mu_{n-1}, & \text { if } x_{n}=x_{n-1}, \\
n \geq 1 .
\end{array}\right.
$$

Firstly, we show that the sequence $\left(x_{n}\right)$ is well defined. Noting strong monotonicity of $F, x_{1} \neq x_{0}$ implies that $F x_{1} \neq F x_{0}$ and $\mu_{1}$ is well defined via the first formula of (40). Consequently, $\mu_{n}(n \geq 2)$ is well defined inductively according to (40) and thus the sequence $\left(x_{n}\right)$ is also well defined using (39).

Next, we estimate $\left(\mu_{n}\right)$ roughly. If $x_{n} \neq x_{n-1}$ (i.e., $F x_{n} \neq$ $\left.F x_{n-1}\right)$, set

$$
\kappa_{n}=\frac{\left\|F x_{n}-F x_{n-1}\right\|}{\left\|x_{n}-x_{n-1}\right\|} .
$$

Observing the fact that $\left(x_{n}\right) \subset S\left(x_{0}, r\right)$ from (39), it turns out that

$$
\eta \leq \frac{\left|\left\langle F x_{n}-F x_{n-1}, x_{n}-x_{n-1}\right\rangle\right|}{\left\|x_{n}-x_{n-1}\right\|^{2}} \leq \frac{\left\|F x_{n}-F x_{n-1}\right\|}{\left\|x_{n}-x_{n-1}\right\|}=\kappa_{n} \leq \kappa_{r} .
$$

Consequently

$$
\frac{\eta}{\kappa_{r}^{2}} \leq \mu_{n}=\frac{\eta}{\kappa_{n}^{2}} \leq \frac{1}{\eta}
$$

By the definition of $\left(\mu_{n}\right)$, we can assert that (43) holds for all $n \geq 1$.

Now we analyze the strong convergence of Algorithm 12.

Theorem 13. Assume that $\lambda_{n} \rightarrow 0(n \rightarrow \infty)$ and $\sum_{n=1}^{+\infty} \lambda_{n}=$ $+\infty$. Then the sequence $\left(x_{n}\right)$ generated by Algorithm 12 converges strongly to the unique solution $x^{*}$ of $\operatorname{VI}(C, F)$.

Proof. Obviously, $\left(x_{n}\right)$ is bounded and so is $\left(F x_{n}\right)$. Setting $\gamma_{n}=\lambda_{n} \mu_{n}$ and $\beta_{n}=(1 / 2)\left(2 \eta-\gamma_{n} \kappa_{r}^{2}\right)$, observing $\lambda_{n} \rightarrow 0$ and 
(43), it is concluded that there exists some positive integer $n_{0}$ such that

$$
0<\gamma_{n}<\frac{\eta}{\kappa_{r}^{2}}, \quad n \geq n_{0},
$$

and consequently

$$
\frac{1}{2} \eta \leq \beta_{n} \leq \eta, \quad n \geq n_{0}
$$

Using Lemma 2, we have from (44) that, for all $n \geq n_{0}, I-$ $\gamma_{n} F: S\left(x_{0}, r\right) \rightarrow H$ is a contraction with coefficient $1-\gamma_{n} \beta_{n}$. This concludes that, for all $n \geq n_{0}$,

$$
\begin{aligned}
&\left\|x_{n+1}-x^{*}\right\|^{2} \\
&=\left\|P_{S\left(x_{0}, r\right)} P_{C_{n}}\left(I-\gamma_{n} F\right) x_{n}-P_{S\left(x_{0}, r\right)} P_{C_{n}} x^{*}\right\|^{2} \\
& \leq\left\|\left(I-\gamma_{n} F\right) x_{n}-\left(I-\gamma_{n} F\right) x^{*}-\gamma_{n} F x^{*}\right\|^{2} \\
& \leq\left(1-\gamma_{n} \beta_{n}\right)\left\|x_{n}-x^{*}\right\|^{2}-2 \gamma_{n}\left\langle F x^{*}, x_{n}-x^{*}-\gamma_{n} F x_{n}\right\rangle . \\
& \leq\left(1-\gamma_{n} \beta_{n}\right)\left\|x_{n}-x^{*}\right\|^{2}-2 \gamma_{n}\left\langle F x^{*}, x_{n}-x^{*}\right\rangle \\
&+2 \gamma_{n}^{2}\left\|F x^{*}\right\|\left\|F x_{n}\right\| .
\end{aligned}
$$

By an argument similar to getting (27)-(29), we have

$$
\begin{aligned}
\left\|x_{n+1}-x^{*}\right\|^{2} \leq & \left\|x_{n}-x^{*}\right\|^{2}-\left\|x_{n}-P_{C_{n}} x_{n}\right\|^{2} \\
& -\left\|P_{C_{n}} x_{n}-P_{S\left(x_{0}, r\right)} P_{C_{n}} x_{n}\right\|^{2}+\gamma_{n} M,
\end{aligned}
$$

where $M$ is a positive constant, which has nothing to do with $n$. Setting

$$
\begin{gathered}
s_{n}=\left\|x_{n}-x^{*}\right\|^{2}, \\
\delta_{n}=-\frac{2}{\beta_{n}}\left\langle F x^{*}, x_{n}-x^{*}\right\rangle+\frac{2 \gamma_{n}}{\beta_{n}}\left\|F x^{*}\right\|\left\|F x_{n}\right\|, \\
\sigma_{n}=\left\|x_{n}-P_{C_{n}} x_{n}\right\|^{2}+\left\|P_{C_{n}} x_{n}-P_{S\left(x_{0}, r\right)} P_{C_{n}} x_{n}\right\|^{2}, \\
\alpha_{n}=M \gamma_{n},
\end{gathered}
$$

then (46) and (47) can be rewritten as the following forms, respectively:

$$
\begin{gathered}
s_{n+1} \leq\left(1-\gamma_{n} \beta_{n}\right) s_{n}+\gamma_{n} \beta_{n} \delta_{n}, \\
s_{n+1} \leq s_{n}-\sigma_{n}+\alpha_{n} .
\end{gathered}
$$

Clearly, $\lambda_{n} \rightarrow 0$ and $\sum_{n=1}^{\infty} \lambda_{n}=\infty$, together with (43)-(45), imply that $\alpha_{n} \rightarrow 0$ and $\sum_{n=1}^{\infty} \gamma_{n} \beta_{n}=\infty$.

By an argument very similar to the proof of Theorem 10, it is not difficult to verify that

$$
\lim _{k \rightarrow \infty} \sigma_{n_{k}}=0
$$

implies

$$
\limsup _{k \rightarrow \infty} \delta_{n_{k}} \leq 0
$$

for any subsequence $\left(n_{k}\right) \subset(n)$. Thus we can complete the proof by using Lemma 8 .

Similar to Algorithm 9, if $P_{C}$ is easy to be calculated, then $P_{C_{n}}$ in Algorithm 12 can also be replaced with $P_{C}$ and it is easy to see that the whole proof of Theorem 13 is valid for this case. The following result similar to Corollary 11 also holds.

Corollary 14. Assume that $\lambda_{n} \rightarrow 0(n \rightarrow \infty), \sum_{n=1}^{+\infty} \lambda_{n}=$ $+\infty$, and $C=H$. Then the sequence $\left(x_{n}\right)$ generated by algorithm

$$
x_{n+1}=P_{S\left(x_{0}, r\right)}\left(I-\lambda_{n} \mu_{n} F\right) x_{n}, \quad n \geq 0,
$$

where $\mu_{n}$ is given as in (40), converges strongly to the unique solution $x^{*}$ of the operator equation $F x=0$.

Finally, we give an iterative algorithm for solving a class $\operatorname{VI}(C, F)$, in which the closed convex subset $C$ is the intersection of finite level sets of convex functions given as follows:

$$
C=\bigcap_{i=1}^{m}\left\{x \in H: c_{i}(x) \leq 0\right\}
$$

where $m$ is a positive integer and $c_{i}: H \rightarrow \mathbb{R}(i=1, \ldots, m)$ is a convex function. We always assume that $c_{i}(i=1, \ldots, m)$ is subdifferentiable on $H$ and $\partial c_{i}(i=1, \ldots, m)$ is bounded operator (i.e., bounded on bounded sets).

Without loss of generality, we will consider only the case $m=2$; that is, $C=C^{1} \cap C^{2}$, where

$$
C^{1}=\left\{x \in H: c_{1}(x) \leq 0\right\}, \quad C^{2}=\left\{x \in H: c_{2}(x) \leq 0\right\} .
$$

Algorithm 15. Choose an arbitrary initial guess $x_{0} \in C$ and an arbitrary element $x_{1} \in S\left(x_{0}, r\right)$ such that $x_{1} \neq x_{0}$. Assume that the $n$th iterate $x_{n}(n \geq 1)$ has been constructed. Continue and calculate the $(n+1)$ th iterate $x_{n+1}$ via the formula

$$
x_{n+1}=P_{S\left(x_{0}, r\right)} P_{C_{n}^{2}} P_{C_{n}^{1}}\left(I-\lambda_{n} \mu_{n} F\right) x_{n}, \quad n \geq 1,
$$

where the sequence $\left(\lambda_{n}\right)$ is in $(0,1), r$ is a constant such that $r>\left\|F x_{0}\right\| / \eta$, the sequence $\left(\mu_{n}\right)$ is given as in $(40)$, and $C_{n}^{i}(i=$ $1,2)$ is determined via the relation

$$
\begin{gathered}
C_{n}^{1}=\left\{x \in H: c_{1}\left(x_{n}\right) \leq\left\langle\xi_{n}^{1}, x_{n}-x\right\rangle\right\}, \\
C_{n}^{2}=\left\{x \in H: c_{2}\left(P_{C_{n}^{1}} x_{n}\right) \leq\left\langle\xi_{n}^{2}, P_{C_{n}^{1}} x_{n}-x\right\rangle\right\} .
\end{gathered}
$$

By an argument similar to the proof of Theorem 13 (together with the proof of Theorem 3.4 of [22]), we have the following result.

Theorem 16. Assume that $\lambda_{n} \rightarrow 0(n \rightarrow \infty)$ and $\sum_{n=1}^{+\infty} \lambda_{n}=$ $+\infty$. Then the sequence $\left(x_{n}\right)$ generated by Algorithm 15 converges strongly to the unique solution $x^{*}$ of $V I(C, F)$, where $C=C^{1} \cap C^{2}$ and $C^{i}(i=1,2)$ is given as in $(55)$. 


\section{Conflict of Interests}

The authors declare that they have no competing interests.

\section{Authors' Contribution}

All authors contributed equally to the writing of this paper.

\section{Acknowledgments}

The authors would like to thank the three referees for their comments and suggestions on improving an earlier version of this paper. This work was supported by the Fundamental Research Funds for the Central Universities (3122014K010) and in part by the Foundation of Tianjin Key Lab for Advanced Signal Processing.

\section{References}

[1] G. Stampacchia, "Formes bilineaires coercivites sur les ensembles convexes," Comptes Rendus de l'Académie des Sciences, vol. 258, pp. 4413-4416, 1964.

[2] C. Baiocchi and A. Capelo, Variational and Quasi Variational Inequalities, John Wiley \& Sons, New York, NY, USA, 1984.

[3] A. Bnouhachem, "A self-adaptive method for solving general mixed variational inequalities," Journal of Mathematical Analysis and Applications, vol. 309, no. 1, pp. 136-150, 2005.

[4] H. Brezis, Operateurs Maximaux Monotone et Semigroupes de Contractions dans les Espace d'Hilbert, North-Holland, Amsterdam, The Netherlands, 1973.

[5] R. W. Cottle, F. Giannessi, and J. L. Lions, Variational Inequalities and Complementarity Problems: Theory and Applications, John Wiley \& Sons, New York, NY, USA, 1980.

[6] M. Fukushima, "Equivalent differentiable optimization problems and descent methods for asymmetric variational inequality problems," Mathematical Programming, vol. 53, no. 1-3, pp. 99-110, 1992.

[7] K. Geobel and S. Reich, Uniform Convexity, Nonexpansive Mappings, and Hyperbolic Geometry, Marcel Dekker, New York, NY, USA, 1984.

[8] F. Giannessi, A. Maugeri, and P. M. Pardalos, Equilibrium Problems: Nonsmooth Optimization and Variational Inequality Models, Kluwer Academic Press, Dordrecht, The Netherlands, 2001.

[9] R. Glowinski, J.-L. Lions, and R. Tremoliers, Numerical Analys is of Variational Inequalities, North Holland, Amsterdam, The Netherlands, 1981.

[10] P. T. Harker and J.-S. Pang, "Finite-dimensional variational inequality and nonlinear complementarity problems: a survey of theory, algorithms and applications," Mathematical Programming, vol. 48, no. 2, pp. 161-220, 1990.

[11] B. S. He, "A class of implicit methods for monotone variational inequalities," Reports of the Institute of Mathematics 95-1, Nanjing University, Nanjing, China, 1995.

[12] B. S. He and L. Z. Liao, "Improvements of some projection methods for monotone nonlinear variational inequalities," Journal of Optimization Theory and Applications, vol. 112, no. 1, pp. 111-128, 2002.

[13] B.-S. He, Z.-H. Yang, and X.-M. Yuan, "An approximate proximal-extragradient type method for monotone variational inequalities," Journal of Mathematical Analysis and Applications, vol. 300, no. 2, pp. 362-374, 2004.

[14] S. N. He and H.-K. Xu, "Variational inequalities governed by boundedly Lipschitzian and strongly monotone operators," Fixed Point Theory, vol. 10, no. 2, pp. 245-258, 2009.

[15] D. Kinderlehrer and G. Stampacchia, An Introduction to Variational Inequalities and Their Applications, vol. 31 of Classics in Applied Mathematics, SIAM, Philadelphia, Pa, USA, 2000.

[16] J.-L. Lions and G. Stampacchia, "Variational inequalities," Communications on Pure and Applied Mathematics, vol. 20, pp. $493-$ 519, 1967.

[17] H. K. Xu and T. H. Kim, "Convergence of hybrid steepestdescent methods for variational inequalities," Journal of Optimization Theory and Applications, vol. 119, no. 1, pp. 185-201, 2003.

[18] H.-K. Xu, "Viscosity approximation methods for nonexpansive mappings," Journal of Mathematical Analysis and Applications, vol. 298, no. 1, pp. 279-291, 2004.

[19] H. Yang and M. G. H. Bell, "Traffic restraint, road pricing and network equilibrium," Transportation Research Part B: Methodological, vol. 31, no. 4, pp. 303-314, 1997.

[20] I. Yamada, "The hybrid steepest-descent method for variational inequality problems over the intersection of the fixed point sets of nonexpansive mappings," in Inherently Parallel Algorithms in Feasibility and Optimization and Their Applications, D. Butnariu, Y. Censor, and S. Reich, Eds., pp. 473-504, NorthHolland, Amsterdam, The Netherlands, 2001.

[21] M. Fukushima, "A relaxed projection method for variational inequalities," Mathematical Programming, vol. 35, no. 1, pp. 5870, 1986.

[22] S. N. He and C. P. Yang, "Solving the variational inequality problem defined on intersection of finite level sets," Abstract and Applied Analysis, vol. 2013, Article ID 942315, 8 pages, 2013.

[23] K. Goebel and W. A. Kirk, Topics on Metric Fixed Point Theory, Cambridge University Press, Cambridge, UK, 1990.

[24] Q. Yang, "The relaxed CQ algorithm solving the split feasibility problem," Inverse Problems, vol. 20, no. 4, pp. 1261-1266, 2004.

[25] G. Lopez, V. Martin-Marquez, F. H. Wang, and H. K. Xu, "Solving the split feasibility problem without prior knowledge of matrix norms," Inverse Problems, vol. 28, no. 8, Article ID 085004, 2012. 


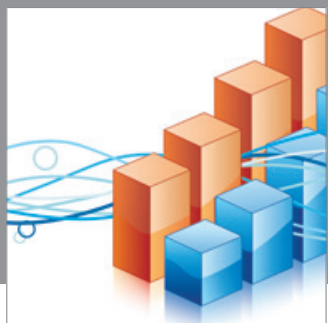

Advances in

Operations Research

mansans

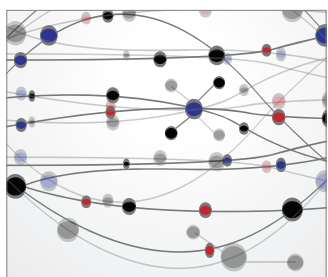

The Scientific World Journal
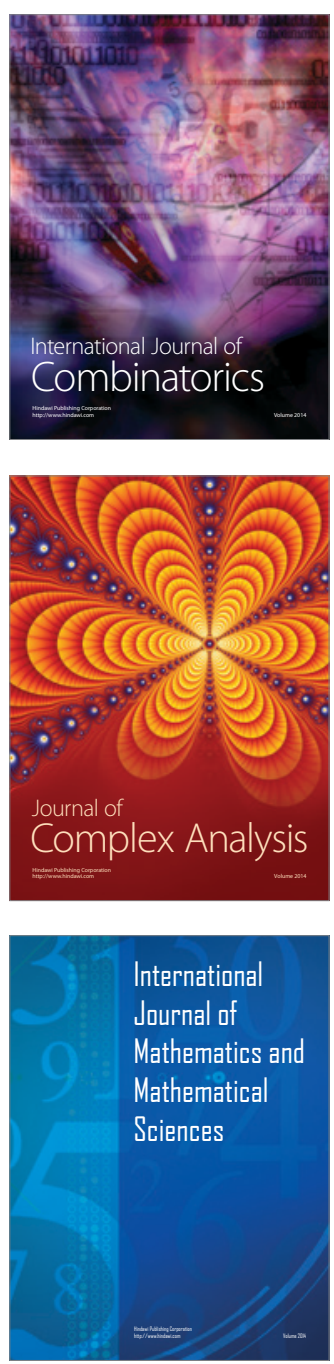
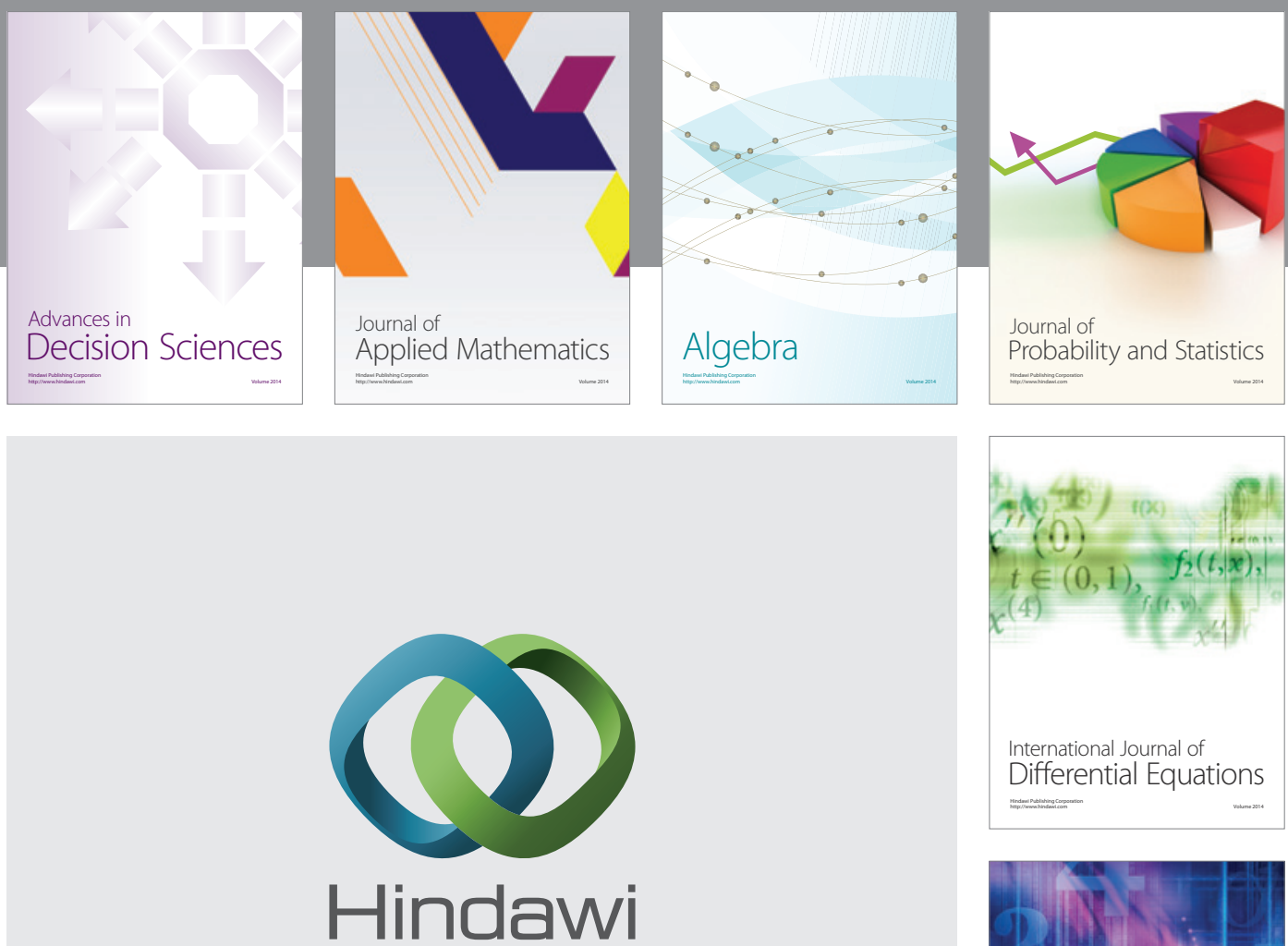

Submit your manuscripts at http://www.hindawi.com
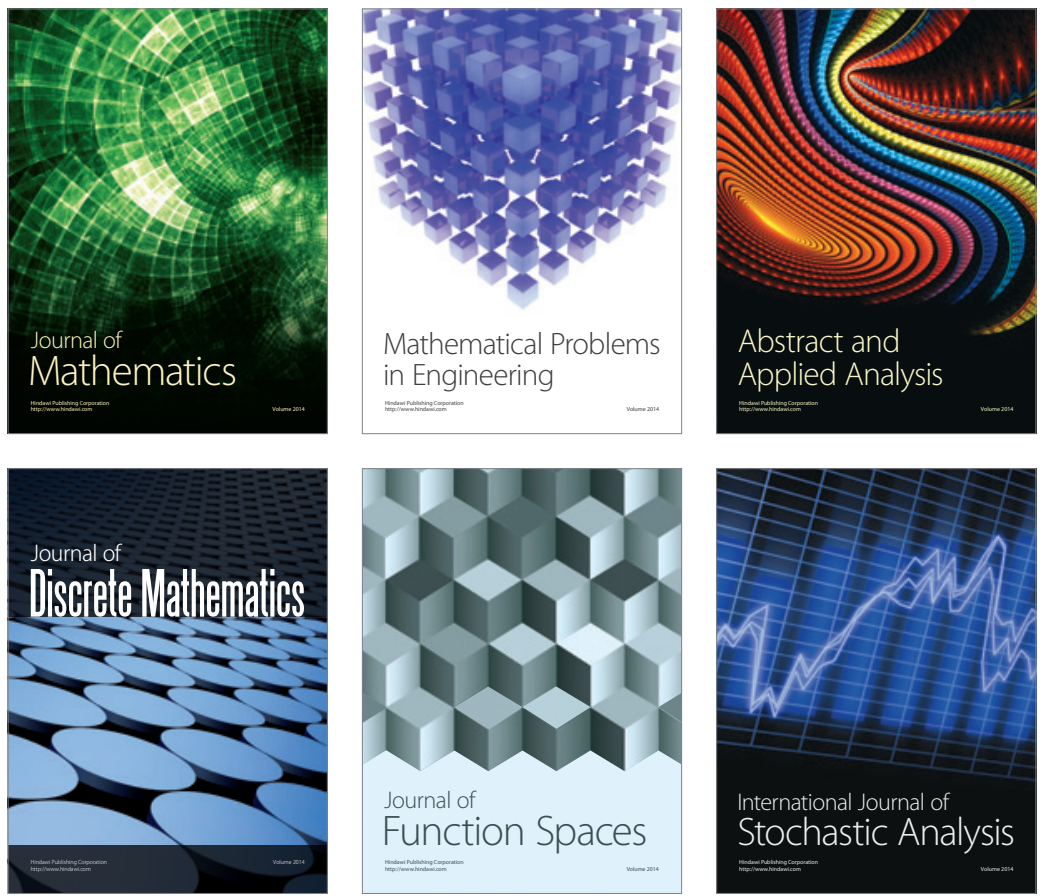

Journal of

Function Spaces

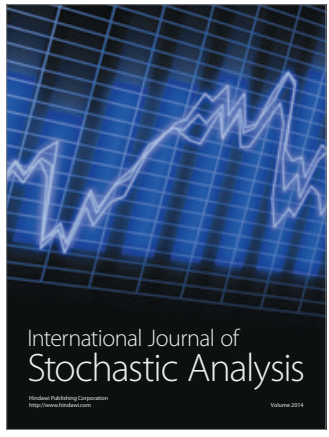

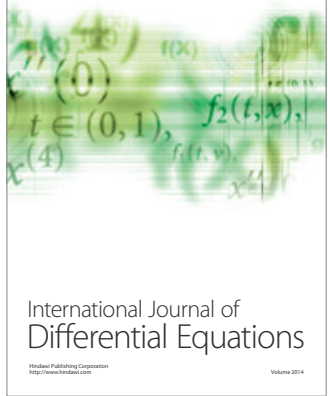
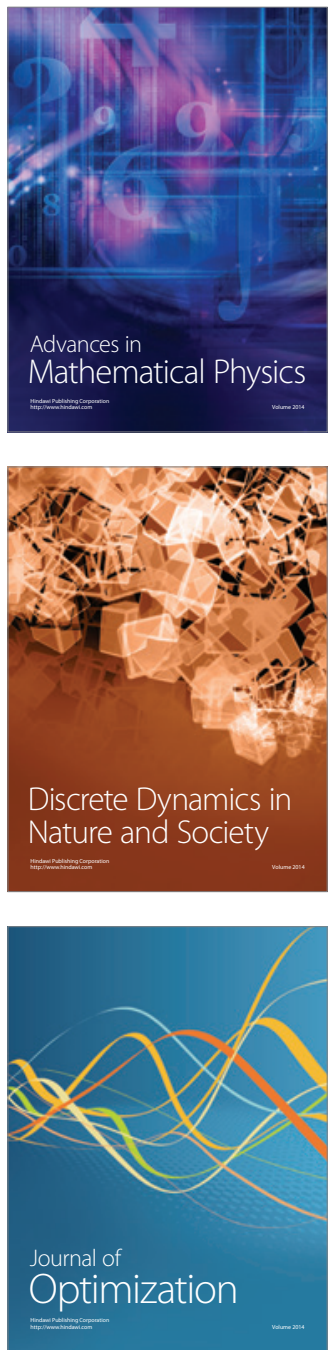